\title{
Electrical characteristics of amyloid beta peptides in vertical junctions
}

\author{
Sohyeon Seo $\mathbb{1}^{1,2}$, Jinju Lee ${ }^{1}$, Jungsue Choi ${ }^{1}$, G. Hwan Park ${ }^{1,3}$, Yeseul Hong ${ }^{1,3}$ and Hyoyoung Lee $\mathbb{B}^{1,2,3,4}$
}

\begin{abstract}
Assembled amyloid beta $(A \beta)$ peptides have been considered pathological assemblies involved in human brain diseases, and the electron transfer or electron transport characteristics of $A \beta$ are important for the formation of structured assemblies. Here, we report the electrical characteristics of surface-assembled A $\beta$ peptides similar to those observed in Alzheimer's patients. These characteristics correlate to their electron transfer characteristics. Electrical current-voltage plots of $A \beta$ vertical junction devices show the $A \beta$ sequence dependence of the current densities at both $A \beta$ monomers (mono-A $A s$ ) and $A \beta$ oligomers (oli-A $A s$ ), while $A \beta$ sequence dependence is not clearly observed in the electrical characteristics of $A \beta$ planar field effect transistors (FETs). In particular, surface oligomerization of $A \beta$ peptides drastically decreases the activity of electron transfer, which presents a change in the electron transport pathway in the $A \beta$ vertical junctions. Electron transport at oli-A $\beta$ junctions is symmetric (tunneling/tunneling) due to the weak and voltage-independent coupling of the less redox-reactive oli-A $\beta$ to the contacts, while that at mono-A $\beta$ junctions is asymmetric (hopping/tunneling) due to redox levels of mono-A $\beta$ voltage-dependently coupled with contact electrodes. Consequently, through vertical junctions, the sequence- and conformation-dependent electrical characteristics of Aßs can reveal their electron transfer activities.
\end{abstract}

\section{Introduction}

Electron transfer and electron transport processes of proteins are important for understanding and gaining insight into their biological functions such as enzymatic reactions ${ }^{1,2}$. However, it is difficult to predict the mechanism of electron transport in proteins due to the size of the protein containing a number of amino acid residues. In biomolecular junctions composed of target biomolecules incorporated between two electrodes, longrange electron transport processes through biomolecules were found to be strongly influenced by molecular size and coupling effects between molecules and electrodes ${ }^{3,4}$. In addition, junction configurations can directly affect the transport efficiency across molecules with regard to

Correspondence: Sohyeon Seo (sshyeon@skku.edu) or

Hyoyoung Lee (hyoyoung@skku.edu)

'Department of Chemistry, Sungkyunkwan University (SKKU), Suwon 16419, Republic of Korea

${ }^{2}$ Creative Research Institute (CRI), Sungkyunkwan University (SKKU), Suwon 16419, Republic of Korea

Full list of author information is available at the end of the article the presence of molecular redox activity ${ }^{5-7}$. For example, junction conductance across proteins is influenced by the electron transport efficiency of interactions between redox-active residues and electrodes ${ }^{7}$. Thus, electron transport behaviors across protein junctions could be involved in structural changes, regardless of whether proteins contain redox-active residues. This behavior is important to consider when studying the mechanical structural changes that cause certain proteins to become toxic.

Amyloid beta $(A \beta)$ peptide molecules are critical components of Alzheimer's disease ${ }^{8-10}$. Concentration- or time-dependent morphological changes that occur as a result of fibrillization via oligomerization of $A \beta$ monomers (mono-A $\beta \mathrm{s})$ during aggregation have been intensively investigated ${ }^{11-14}$. Moreover, electronic conductance studies of $\mathrm{A} \beta$ peptide structures have revealed the importance of structural parameters such as the sequences and assembly conditions of $A \beta^{15,16}$. However, understanding how mechanical pathway-dependent chemical

\section{(c) The Author(s) 2021}

(c) Open Access This article is licensed under a Creative Commons Attribution 4.0 International License, which permits use, sharing, adaptation, distribution and reproduction cc) in any medium or format, as long as you give appropriate credit to the original author(s) and the source, provide a link to the Creative Commons license, and indicate if changes were made. The images or other third party material in this article are included in the article's Creative Commons license, unless indicated otherwise in a credit line to the material. If material is not included in the article's Creative Commons license and your intended use is not permitted by statutory regulation or exceeds the permitted use, you will need to obtain permission directly from the copyright holder. To view a copy of this license, visit http://creativecommons.org/licenses/by/4.0/. 


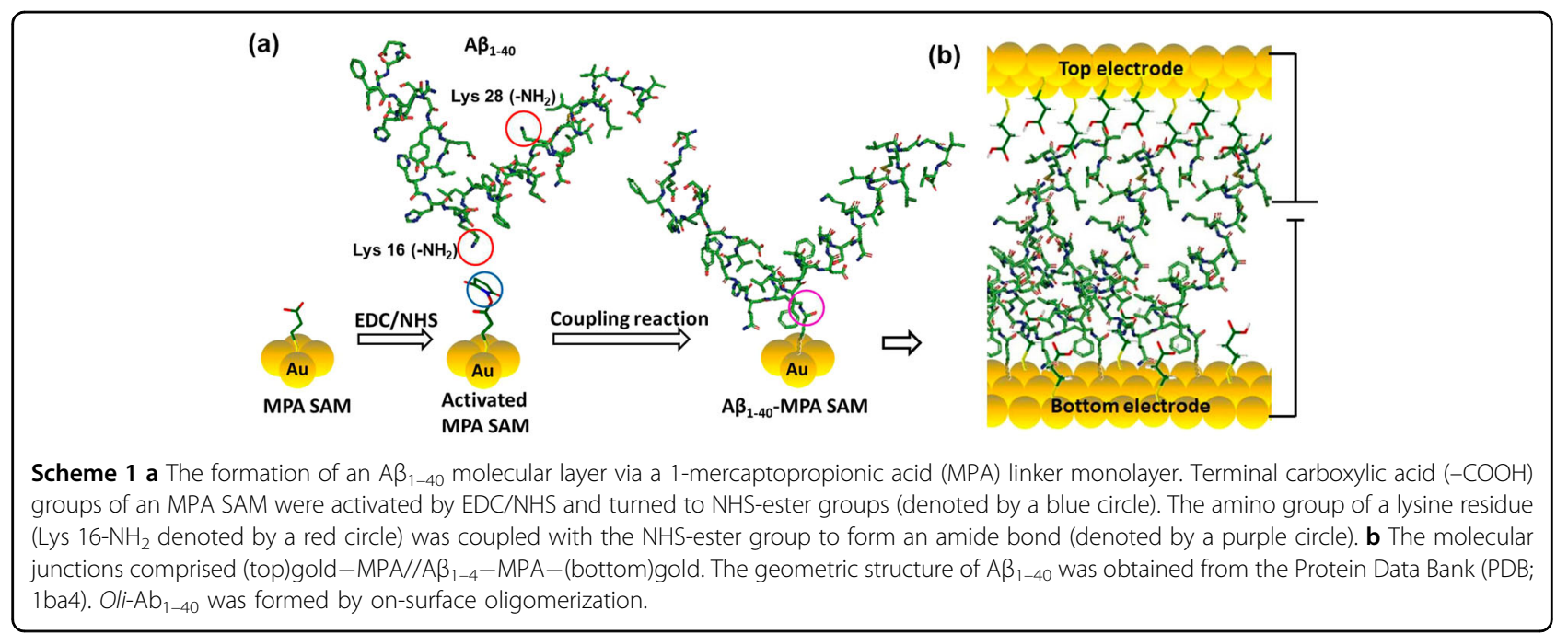

activity (or toxicity) results from oligomerization of $A \beta$ remains a significant challenge, although oligomers and fibrils are known to play an active role ${ }^{17-19}$. Based on previous reports ${ }^{11,15,20}$, electron transport pathways of amyloid-based proteins whose structural conformation can be determined by a mechanical transition from monomers to oligomers result in changes in chemical activity, such as the redox activity of amino acid residues. Thus, oligomerization of $A \beta$ could induce a change in electron transport across structurally altered $A \beta$ molecules (e.g., $\mathrm{A} \beta$ oligomers, oli- $\mathrm{A} \beta \mathrm{s}$ ) on a surface. The electron transport efficiency of mono- and oli-A $\beta$ s can determine the corresponding mechanical pathways and chemical activities during aggregation processes.

In this work, the electrical characteristics of $A \beta$ peptides were presented to describe the conformational status of the peptides. Surface-immobilized A $\beta$ peptide layers (e.g., $A \beta$-linker-gold or $A \beta$-linker/graphene (G)) were used. Electron transport pathways through $A \beta$ were investigated by using three $A \beta$ peptides (e.g., $A \beta_{1-40}, A \beta_{1-28}$, and $A \beta_{12-28}$ ) in vertical gold/biomolecule-gold junctions ("/" denotes physical contact and "-" denotes chemical contact). Electrical characteristics of the vertical junctions showed $A \beta$ sequence dependence, while the current flowing in the field effect transistor (FET) with an $A \beta$ peptide/G channel was weakly dependent on the peptide sequences. In particular, the surface oligomerization of the $A \beta$ peptides drastically changed the electron transfer characteristics by shifting the electrochemical potentials associated with the change in chemical activity. This was clearly revealed in the electrical characteristics of $A \beta$ vertical junctions rather than $A \beta / G$ FETs. (1) Redoxactive peptide residues of mono-A $\beta$ s strongly coupled with the linker monolayer led to asymmetric electron transport characteristics. (2) However, oligomeric $A \beta$ junctions exhibited only symmetric electron transport characteristics due to weak redox activity. These findings indicate that the intermolecular assembly of $A \beta$ peptides results in changes in surface chemical activities (i.e., electron transfer activities), leading to changes in the electron transport pathways.

\section{Results and discussion}

To study the electrical characteristics of $A \beta$ peptides through a vertical junction, it is important to have welldefined molecular assembly layers on metal electrodes. However, the formation of molecular layers of proteins or polypeptides might be complicated by a lack of spatial control. Thus, a self-assembled molecular linker monolayer of 1-mercaptopropionic acid (MPA) was used to immobilize $A \beta$ onto a gold electrode, which was activated by coupling reactions with 1-ethyl-3-(3-dimethylamino) propyl carbodiimide/ $N$-hydroxysuccinimide (EDC/NHS) (Scheme 1). Mono-A $\beta$-MPA molecular layers were formed via chemical bonding of lysine (Lys 16 or 28 denoted with red circles) residues onto the activated MPA (EDC/NHS-MPA) molecular layer (denoted by a blue circle). Then, the vertical junction of top gold-MPA//A $\beta$ -MPA-bottom gold was fabricated.

\section{Conformation- and sequence-dependent electron transfer of $A \beta$ peptide-immobilized surfaces \\ Characteristics of in-solution oligomerization}

Changes in the molecular conformation and chemical activity (i.e., electron transfer activity) due to the oligomerization of $A \beta$ molecules were verified by circular dichroism (CD) spectroscopy and differential pulse voltammetry (DPV). In Fig. 1a, the CD spectrum of mono$\mathrm{A} \beta_{1-40}$ solution showed a characteristic absorption minimum of unstructured monomers at approximately $200 \mathrm{~nm}^{19,21}$. In-solution oligomerization under our conditions ( $1 \mathrm{~h}$ incubation) resulted in a shift in the absorption 

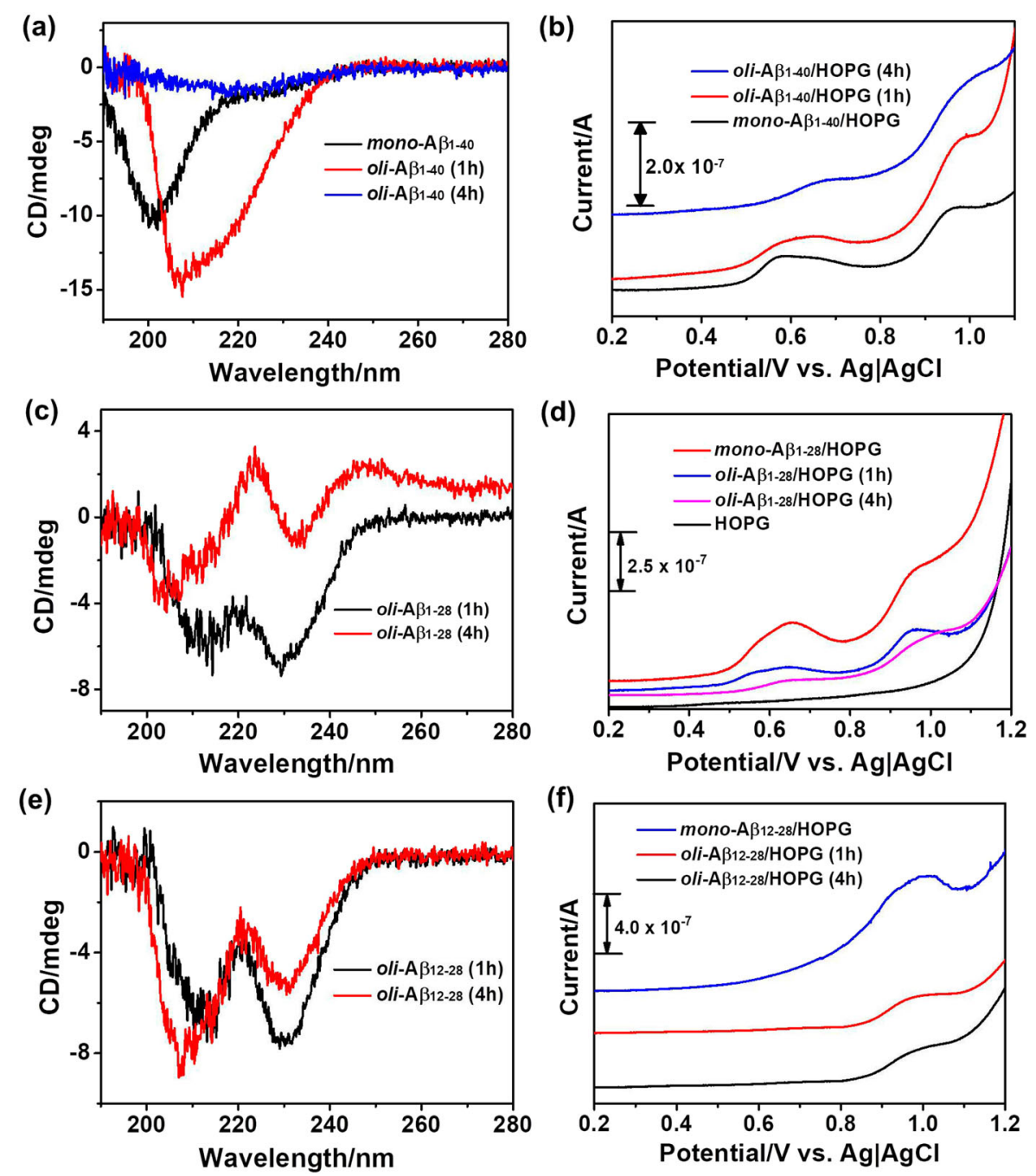

Fig. 1 In-solution oligomerization. a Circular dichroism (CD) spectra of mono- and oli-A $\beta_{1-40}$ solutions according to the incubation period. b Differential pulse voltammograms (DPVs) of mono- and oli-A $\beta_{1-40}$ physically adsorbed HOPG electrodes. c CD spectra of the oli-A $\beta_{1-28}$ solution according to the incubation period. $\mathbf{d}$ DPVs of $A \beta_{1-28}$ physically adsorbed HOPG electrodes. e CD spectra of the oli-A $\beta_{12-28}$ solution according to the incubation period. $\mathbf{f}$ DPVs of $A \beta_{12-28}$ physically adsorbed on HOPG electrodes. CD was measured at $25^{\circ} \mathrm{C}$. DPV was measured in $0.1 \mathrm{M}$ phosphate buffer solution at a scan rate of $2 \mathrm{mV} \mathrm{s}^{-1}$.

minimum to approximately $210 \mathrm{~nm}$ due to the adoption of a secondary structural conformation ${ }^{19}$, indicating oligomeric aggregation of the mono-A $\beta$ s. After $4 \mathrm{~h}$ of incubation, the CD signal was drastically decreased. In addition, $\mathrm{CD}$ spectra characterized by two negative peaks for both oli- $\mathrm{A} \beta_{1-28}$ and oli-A $\beta_{12-28}$ also indicated that the oli- $\mathrm{A} \beta \mathrm{s}$ had adopted a secondary structural conformation in short $A \beta_{1-28}$ and $A \beta_{12-28}$ peptides (Fig. 1c, e) ${ }^{19}$. The oligomerization effects of mono-A $\beta$ s on electron transfer behaviors were explored by electrochemical responses of physically immobilized $\mathrm{A} \beta$ peptides of mono- $\mathrm{A} \beta$ and in-solution oligomerized oli-A $\beta$ on highly oriented pyrolytic graphite (HOPG) electrodes (Fig. 1b, d, f $)^{22,23}$. The $A \beta_{1-40}$ peptide contains electrochemically active functional groups (e.g., histidine (His 6,13,14), tyrosine (Tyr 10), and methionine (Met 35)) that can be oxidized in the potential range from 0.56 to $0.96 \mathrm{~V}_{\mathrm{Ag} \mid \mathrm{AgCl}}$ on a glassy carbon (GC) electrode ${ }^{22,23}$. In Fig. $1 \mathrm{~b}$, the oxidative peaks of $\mathrm{Tyr}$ at approximately $0.56 \mathrm{~V}_{\mathrm{Ag} \mid \mathrm{AgCl}}$ and His/Met at approximately $1.0 \mathrm{~V}_{\mathrm{Ag} \mid \mathrm{AgCl}}$ were observed for $\mathrm{A} \beta_{1-40}$; these were also observed at approximately $0.56 \mathrm{~V}_{\mathrm{Ag} \mid \mathrm{AgCl}}$ for $\mathrm{Tyr}$ and approximately $0.96-1.0 \mathrm{~V}_{\mathrm{Ag} \mid \mathrm{AgCl}}$ for His for $\mathrm{A} \beta_{1-28}$ (Fig. 1d), while an oxidative His peak was only observed at approximately $1.0 \mathrm{~V}_{\mathrm{Ag} \mid \mathrm{AgCl}}$ for $\mathrm{A} \beta_{12-28}$ (Fig. 1f). Electrochemically active residues buried by the aggregation of monomers into oligomers can lead to a decrease in electrochemical activity. Thus, the electron transfer sensitivities of electrochemically active residues in the oli-A $\beta \mathrm{s}$ 

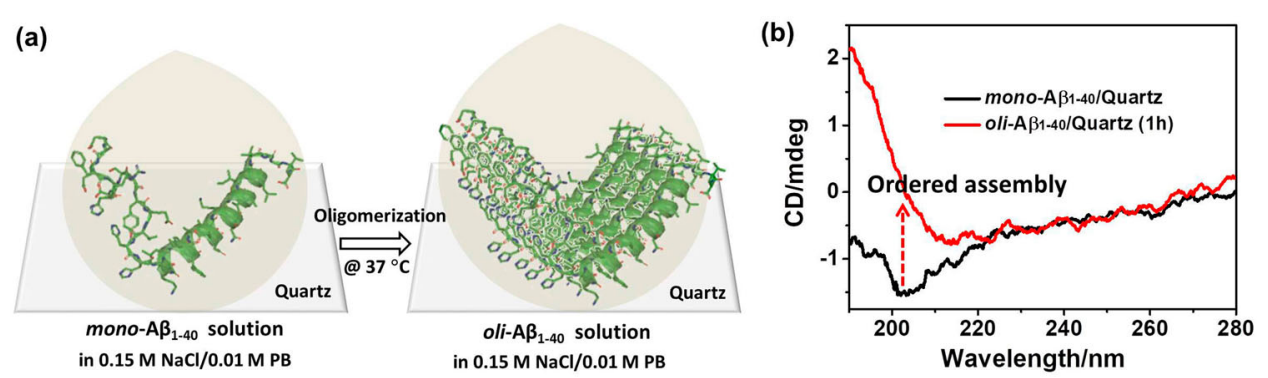

(c)

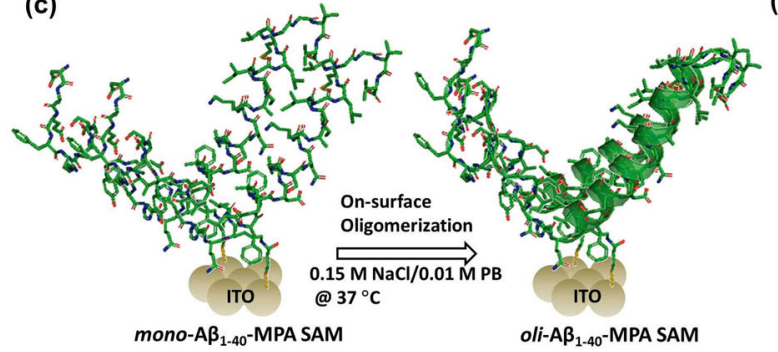

d)

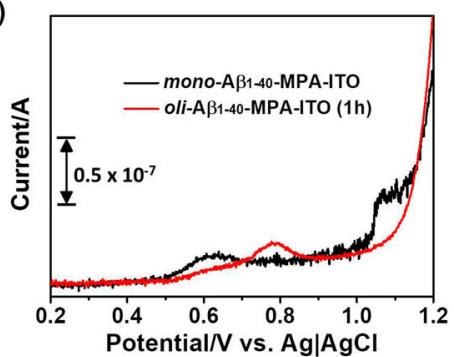

Fig. 2 On-surface oligomerization. a Illustration of on-surface oligomerization of the $A \beta_{1-40}$ molecular layer on a quartz substrate. $\mathbf{b}$ CD spectra of mono- and oli-A $\beta_{1-40}$ physically adsorbed on quartz substrates. c Illustration of surface oligomerization of the $A \beta_{1-40}$ molecular layer chemically bound on an indium tin oxide (ITO)/glass substrate. d DPVs of mono- and oli-A $\beta_{1-40}$ chemically adsorbed on MPA-ITO/glass in 0.1 M phosphate buffer solution at a scan rate of $2 \mathrm{mV} \mathrm{s}^{-1}$.

were decreased, and the peaks shifted to positive potentials. Furthermore, the process of in-solution $A \beta$ oligomerization (or fibrillization via ordered assembly) was verified by scanning electron microscopy (SEM) (Fig. $\mathrm{S} 1 \mathrm{a}-\mathrm{d})$. Apparently, oligomerization of the mono-A $\beta_{1-40}$ solution by $1 \mathrm{~h}$ incubation could not change the morphology of an oli-A $\beta_{1-40}$-deposited surface compared with that of a mono-A $\beta_{1-40}$-deposited surface. After 4 and $24 \mathrm{~h}$ incubations, short protofibrils and long mature fibrils were observed on the oli-A $\beta_{1-40}$-deposited surfaces, respectively.

\section{Characteristics of on-surface oligomerization}

To promote the formation of oli-A $\beta$ on a substrate, the on-surface oligomerization of mono-A $\beta$ was conducted. $\mathrm{A} \beta_{1-40}$ molecules oligomerized on a quartz substrate were assembled into oli-A $\beta_{1-40}$ and physically adsorbed on the substrate (Fig. 2a) for the identification of structural changes. Unlike the oligomerization of $A \beta$ that occurred in solution, the on-surface oligomerization resulted in a drastic CD spectral change where a pair of positive and negative peaks appeared at approximately 190 and $210 \mathrm{~nm}$, characteristic of a beta-sheet structure ${ }^{19}$, indicating the ordered assembly of oli-A $\beta_{1-40}$ (Fig. 2b). This indicates the influence of the surface on the oligomerization of $\mathrm{A} \beta^{24}$; this surface oligomerization is distinguishable from in-solution oligomerization (Fig. 1). Furthermore, for the formation of oli-A $\beta$ molecular layers on an MPA self-assembled monolayer (SAM), the onsurface oligomerization of mono-A $\beta-\mathrm{MPA}$ molecular layers was conducted in $0.01 \mathrm{M}$ phosphate buffer (PB) solution containing $0.15 \mathrm{M} \mathrm{NaCl}$ at $37^{\circ} \mathrm{C}$ for $1 \mathrm{~h}$ (Scheme $1 \mathrm{a}$ and Fig. 2c). Mono-A $\beta$-tethered gold or indium tin oxide (ITO) electrodes were used for electrical and electrochemical measurements, respectively. As shown in Scheme 1a, lysine residues with a charged long chain would be relatively favorable for the coupling reaction with NHS ester compared with the primary amines at the C- or N-termini, which have short chains that can be statically hindered by nearby peptide chains. Unfortunately, CD of the oligomerized amyloid peptide MPA-ITO could not be observed, probably due to insufficient amounts of amyloid peptides on the MPA SAM. In particular, an ITO electrode was suitable for not only the formation of an MPA SAM but also to maintain a stable potential window in $\mathrm{PB}$ electrolyte solution. In Fig. 2d, mono-A $\beta$-tethered ITO electrodes $\left(\mathrm{A} \beta_{1-4}-\mathrm{MPA}-\mathrm{ITO}\right)$ showed a change in electron transfer activities to shift oxidation peaks to approximately 0.6 and $1.05 \mathrm{~V}_{\mathrm{Ag} \mid \mathrm{AgCl}}$, as shown in Fig. 1. This electrochemical activity changed significantly after the oligomerization on the surface was allowed to proceed for $1 \mathrm{~h}$, whereas physically adsorbed oli-A $\beta$ s that were oligomerized in solution showed a gradual decrease in electrochemical activity as incubation time increased (Fig. 1b). Only one peak at approximately $0.8 \mathrm{~V}_{\mathrm{Ag} \mid \mathrm{AgCl}}$ was observed for the oli-A $\beta_{1-4}-\mathrm{MPA}-\mathrm{ITO}$. One of the possible reasons is that electrochemically active residues on the $A \beta$ molecules tethered on the electrodes via chemical binding are probably more hindered by bulky side residues than physically adsorbed $A \beta$ 
molecules. Thus, electron transfer from the electrode could be blocked, even at an early stage of oligomerization (e.g., $1 \mathrm{~h}$ incubation $)^{23}$. The other possible reason could be a change in electron transfer activity due to the strong interaction with the electrode leading to a change in chemical reaction potentials.

Oligomeric changes in $A \beta$ on the surface induced a change in the surface hydrophilicity (Fig. S2), which can change the electrochemical activity of the surface in the electrolyte. Both MPA and EDS/NHS-MPA SAMs are hydrophilic and can interact positively with hydrophilic residues of mono-A $\beta$ s. After modification of the SAMs with $A \beta$ molecules, the surfaces retained their hydrophilicity (Fig. S2a). However, oligomerization of mono-A $\beta$ SAM led to a decrease in surface hydrophilicity (Fig. S2b). This surface change could result in the electrochemical hindrance of oli-A $\beta$ SAMs, leading to the disappearance of electrochemical reactivity. Clean HOPG, on the other hand, exhibited hydrophobicity (Fig. S2c), inducing repulsive interactions with hydrophilic residues of $A \beta$ molecules, which resulted in the hydrophilic surfaces of the physically adsorbed oli-A $\beta$ molecules (Fig. S2d). Thus, the electrochemical activity of $\mathrm{A} \beta$ molecules in oli-A $\beta \mathrm{s} /$ HOPG was retained for a few hours of incubation, unlike that of the A $\beta$ molecules in oli-A $\beta \mathrm{s}-\mathrm{MPA}-\mathrm{ITO}$.

\section{Surface morphology and binding energy of $A \beta$ peptide-MPA monolayers}

In molecular electronic devices, the surface order (or roughness) of molecular layers influences the junction properties $^{25}$. To explore changes in surface morphology by mono-A $\beta$ modification on an MPA SAM and further on-surface oligomerization, mono- or oli-A $\beta_{1-40}$ molecular SAMs were examined by scanning tunneling microscopy (STM) (Fig. 3a-c). STM images were used to evaluate the surface morphology in nanoscale molecular layers on a single-crystal electrode. The etch pits formed during thiol adsorption on $\mathrm{Au}$ (111) are shown. Surface roughness increased in both mono- and oli-A $\beta$ molecular layers, indicating that the surface ordering decreased with the formation of $A \beta$ layers compared with that of the MPA layer. Features from defects in the mono- or oli-A $\beta$ molecular layers were distinguishable from those in the MPA SAM, indicating the formation of new defects in the $A \beta$ layers. The surface morphology of the $A \beta$ molecular layers was slightly changed by oligomerization. These observations indicated that the oli-A $\beta$ molecular layers maintained the ordering of mono- $\mathrm{A} \beta$ molecular layers after oligomerization, which also revealed the advantageous surface conditions of $A \beta$ for the fabrication of molecular layer-based molecular junctions. The microscale surface morphology of different $A \beta$ peptide layers was evaluated by AFM on an e-beam-deposited gold electrode (Fig. S3a-c). The surface roughness was increased by an increase in the molecular length of $A \beta$ peptides. Ellipsometry measurements showed an increase in the film thickness as the molecular length of $A \beta$ peptides increased (Fig. S3d).

Furthermore, the $A \beta$ peptides with the MPA molecular SAM exhibited changes in their binding energies with gold electrodes; these binding energies were verified by ultraviolet photoelectron spectroscopy (UPS) (Fig. 3d,e). Surface modification with the activated-MPA (i.e., (EDS/ NHS)-MPA) SAM led to an increase in binding energies with the electrodes compared to the binding energies of the MPA SAM according to the analysis of the secondary electron cutoff (SEC) region (Fig. 3d). This phenomenon was also observed after modification with mono-A $\beta$ s. No specific correlation between sequences of $A \beta$ peptides and shifts in work function was found in the SEC region (the left plot in Fig. 3e). However, the energy offset between the Fermi level of the gold electrode and the nearest molecular orbital (e.g., highest occupied molecular orbital, HOMO) became larger in the HOMO region as the sequence length increased (the right plot in Fig. 3e). Thus, electron injection barriers from the electrode to the mono- $\mathrm{A} \beta$ layers decreased from mono-A $\beta_{1-40}$ to mono- $\mathrm{A} \beta_{1-28}$ and mono-A $\beta_{12-28}$, which indicates length-dependent electron transport behaviors in those junctions ${ }^{26}$. Moreover, as oligomerization of the $A \beta$ peptides occurred on the surface, the binding energy contributed by chemical contacts became weaker in the SEC region, and the energy offset between the Fermi level of the electrode and the nearest molecular orbital became larger in the HOMO region. Thus, electron transport across the mono-A $\beta$ junction can be favored to a greater extent than that across the oli- $\mathrm{A} \beta$ junction.

\section{Conformation- and sequence-dependent electrical characteristics of $A \beta$ peptides in soil-state devices Electrical characteristics of $A \beta$ vertical junctions in a cross- bar device}

For the fabrication of a cross-bar device, protein stability could be an issue due to nonphysiological environments during the fabrication of molecular junctions, especially contact formation of a top electrode. Soft contacts such as AFM tips ${ }^{3,27,28}$, soft materials ${ }^{6,29}$, or gold nanowires ${ }^{7}$ have therefore been used to study the electron transport mechanisms of proteins. In this work, the vertical junction devices of $A \beta$ were configured into top gold-MPA//A $\beta-\mathrm{MPA}$-bottom gold, where top gold-MPA electrodes were assembled by a soft contact on bottom mono- or oli-A $\beta-\mathrm{MPA}$-gold electrodes (Fig. S4). In our previous work ${ }^{30}$, the electrical characteristics and stability of the vertical molecular junction assembled by soft contact were verified with length-dependent tunneling transport properties for a series of alkanethiol molecules. 
(a)
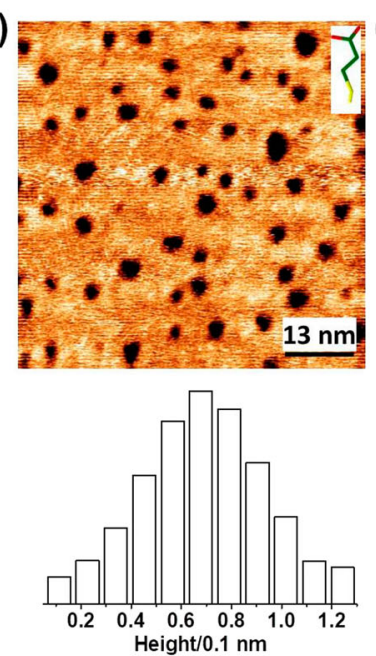

(d)

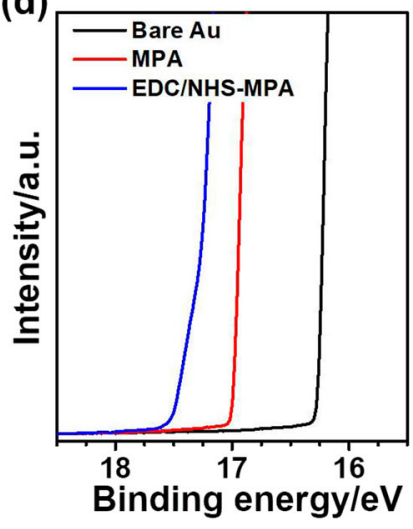

(b)
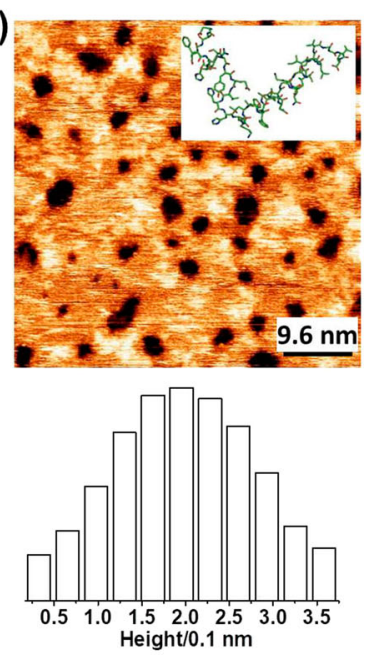

(e)

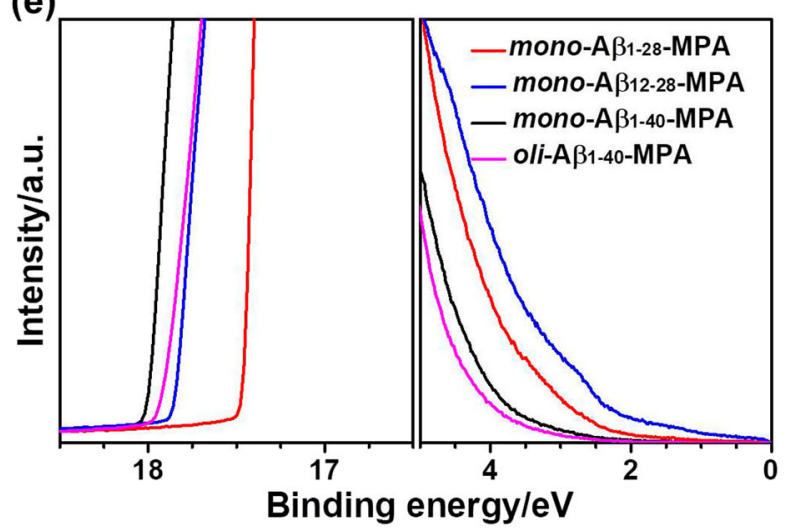

Fig. 3 Changes in surface morphology and binding energy. a-c STM images and histograms of the surface roughness of an MPA SAM, a mono$A \beta_{1-4}-$ MPA SAM, and an oli-A $\beta_{1-4}-$ MPA SAM on Au (111) obtained in $0.1 \mathrm{M} \mathrm{HClO}_{4}$ aqueous solution at $V_{\text {bias }}=0.1 V_{\text {tip }}, V_{\text {sub }}=0.1 V_{\text {SCE, }}$ and $I_{\text {set }}=$ $0.1 \mathrm{nA}$. d, e Ultraviolet photoelectron spectroscopy (UPS) spectra of each molecular layer.

Three different peptides $\left(A \beta_{1-40}, A \beta_{1-28}\right.$, and $\left.A \beta_{12-28}\right)$ formed mono- $\mathrm{A} \beta$ and oli- $\mathrm{A} \beta$ molecular junctions. Plots of current density against voltage $(J / V)$ clearly showed that mono-A $\beta$ molecular junctions had asymmetric characteristics (Fig. 4a, b), while oli-A $\beta$ junctions had symmetric characteristics (Fig. 4d, e). The statistical $J$ curves as a function of applied voltage (with 95\% confidence intervals of the mean) were obtained with 50 devices for each SAM. These are molecular junctions of non-shorted devices, which allowed $>80 \%$ yield. For both mono- and oli-A $\beta$ junctions, molecular lengthdependent $J / V$ plots were obtained, indicating that the junction distance of a smaller $A \beta$ peptide is shorter than that of a larger $A \beta$ peptide. Thus, the $J$ values of $A \beta_{12-28}$ junctions were larger than those of $A \beta_{1-28}$ and $A \beta_{1-40}$ junctions, as expected. For mono-A $\beta$ junctions, the $J / V$ rectifications indicated asymmetric electronic coupling via either chemical or physical contacts of $A \beta$ peptides to the electrode, although compositionally symmetric junctions were fabricated for (top) $\mathrm{Au}-\mathrm{MPA} / \mathrm{A} \beta \mathrm{s}$ -MPA-Au (bottom).

In particular, the asymmetric $J / V$ plots were found to be dependent on the bias voltage value. At a low voltage range $( \pm 0.5 \mathrm{~V})$, all mono- $\mathrm{A} \beta$ junctions had symmetric sigmoidal curves (Fig. S5), while in the voltage range higher than $\pm 1.0 \mathrm{~V}$, asymmetric sigmoidal $J / V$ curves were observed. This bias voltage-dependent asymmetric electron transport indicates current rectification behavior involving the asymmetric electronic structure of mono- $\mathrm{A} \beta$ molecules induced by a high bias voltage ${ }^{31,32}$. Furthermore, the $J$ values decreased in the oligomeric A $\beta$ junctions of (top) $\mathrm{Au}-\mathrm{MPA} / /$ oli-A $\beta-\mathrm{MPA}-\mathrm{Au}$ (bottom). Thus, oligomerization of $A \beta$ peptides in molecular layers can equalize the electronic and/or contact properties in their molecular junctions.

The effects of the asymmetric contacts were examined by using control devices (Fig. S6). Junctions composed of asymmetric contacts, such as (top) gold-MPA//MPA-gold 
(a)

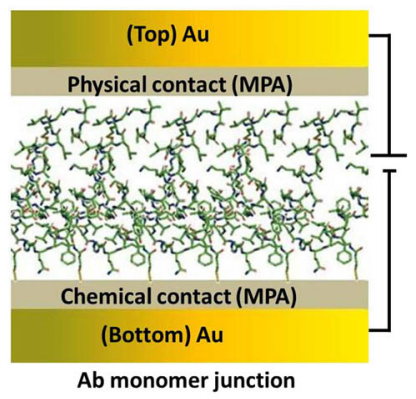

(c)

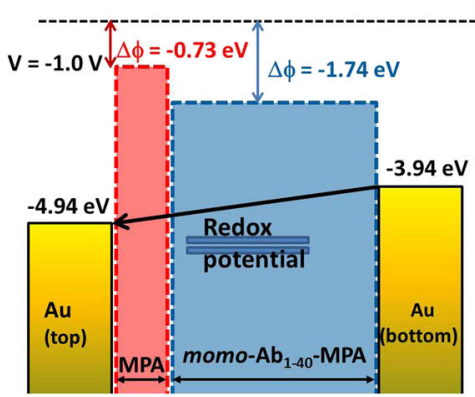

(b)

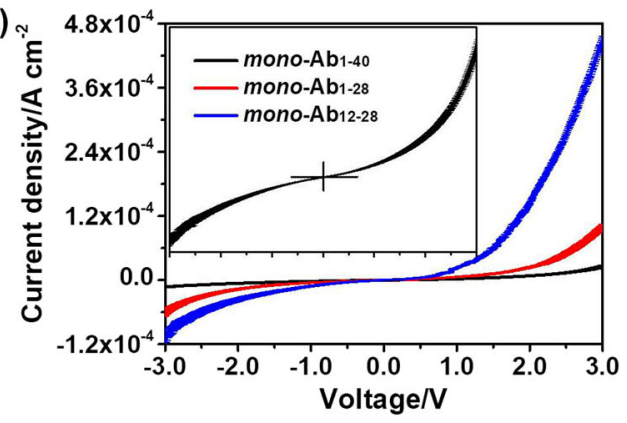

Vacuum

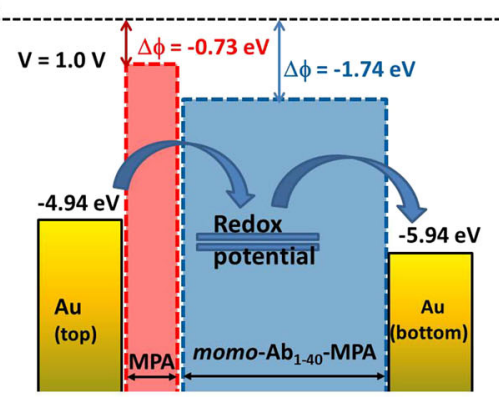

(d)

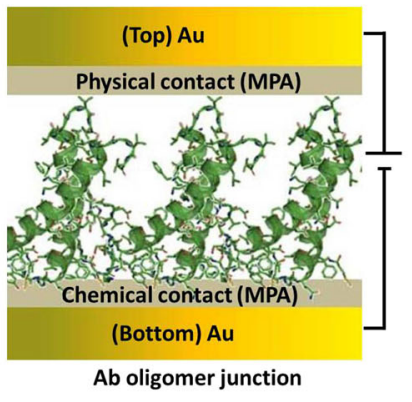

(e)

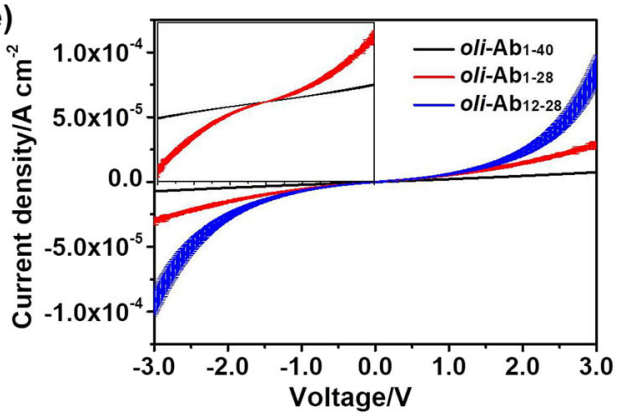

(f)

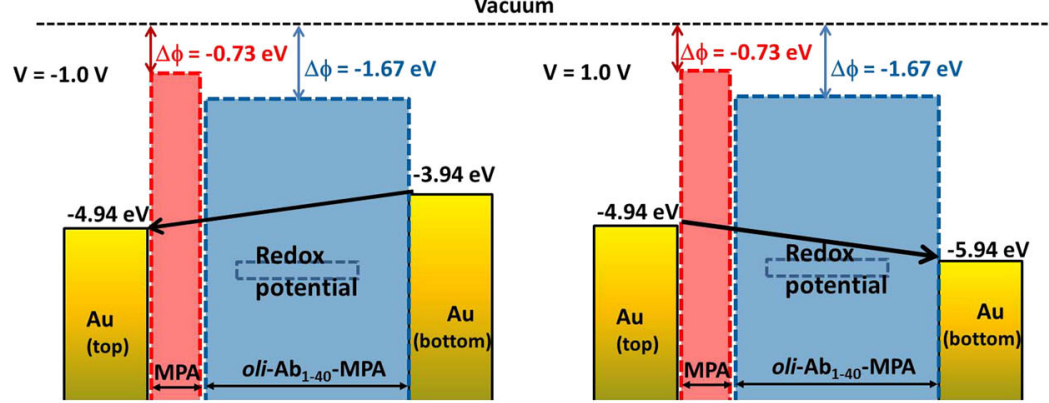

Fig. 4 Electrical characteristics of $\mathbf{A} \boldsymbol{\beta}$ peptides in a vertical junction device. a Illustration of a mono-A $\beta_{1-40}$ vertical junction. $\mathbf{b}$ Current density/ voltage (JM) plots of the $A \beta$ sequence-dependent electron transport characteristics of mono-A $\beta$ molecular junctions. Inset: enlarged plots for mono$A \beta_{1-40}$. The statistical J values with $95 \%$ confidence intervals of the mean were obtained with 50 devices for each SAM (>80\% of device yield). c Proposed mechanisms of electron transport through $A \beta_{1-40}$ molecular junctions with regard to applied bias voltages. The straight arrow at $V=-$ $1.0 \mathrm{~V}$ and the curved arrows at $V=1.0 \mathrm{~V}$ indicate the tunneling (where electrons traverse an energy barrier) and hopping (where electrons traverse localized sites) processes, respectively. The redox levels were obtained from UPS data. $\mathbf{d}$ Illustration of an oli-A $\beta_{1-40}$ vertical junction. e Current density/voltage $(J M$ plots of the $A \beta$ sequence-dependent electron transport characteristics of oli-A $\beta$ molecular junctions. Inset: enlarged plots for oli$A \beta_{1-28}$ and oli-A $\beta_{1-40}$. The statistical $J$ values with $95 \%$ confidence intervals of the mean were obtained with 50 devices for each SAM (>80\% of device yield). $\mathbf{f}$ Proposed mechanisms of electron transport through $A \beta_{1-40}$ molecular junctions with regard to applied bias voltages. The straight arrows at $V=-1.0$ and $1.0 \mathrm{~V}$ indicate the tunneling processes. 
(bottom) and (top) gold//MPA-gold (bottom) junctions without $\mathrm{A} \beta$ molecules, were fabricated. These asymmetric contacts exhibited symmetric behavior in the $J / V$ curves, similar to oli-A $\beta$ molecular junctions, indicating that asymmetric contact junctions do not strongly influence the asymmetric electron transport behavior of mono-A $\beta$ molecules. In general, both (exponentially length-dependent) tunneling and (inversely proportional lengthdependent) hopping processes can contribute to electron transport pathways across molecular junctions ${ }^{29,33,34}$. Based on the molecular size-dependent $J / V$ plots of mono$\mathrm{A} \beta$ molecules (Fig. 4b, e), the electron transport mechanism was distance dependent, although junction distances formed by three $A \beta$ molecules could not be correctly determined. In addition, bias voltage-dependent transport characteristics indicated a redox centerinvolved mechanism ${ }^{26,35}$. The energy level of the redox potential $\left(E_{\mathrm{HOMO}}\right)$ to vacuum can be estimated by using the formal half-wave potential $\left(E_{1 / 2}\right)^{36} ; E_{\mathrm{HOMO}}=E_{\mathrm{abs}, \mathrm{NHE}}$ - $E_{1 / 2, \mathrm{NHE}}$, where $E_{\mathrm{abs}, \mathrm{NHE}}$ is the absolute potential energy of the normal hydrogen electrode, NHE $\left(E_{\mathrm{abs}, \mathrm{NHE}}=\right.$ $-4.44 \mathrm{eV}$ and $\left.E_{\mathrm{Ag} \mid \mathrm{AgCl}}=0.197 E_{\mathrm{NHE}}\right)$. Thus, the energy levels of the redox potentials of mono-A $\beta_{1-40}$ were estimated to be at approximately $-5.25 \mathrm{eV}$ and $-5.69 \mathrm{eV}$, whereas that of oli-A $\beta_{1-40}$ was measured to be only approximately $-5.49 \mathrm{eV}$ (Fig. 2d). In the case of mono-
$\mathrm{A} \beta_{1-40}$, the HOMO energy levels can be arranged between the Fermi levels of gold at $1.0 \mathrm{~V}$, which were depicted at the same location in the junctions in Fig. 4c due to the undefined locations of redox centers. However, one of the HOMO energy levels of oli-A $\beta_{1-40}$ can be arranged between the Fermi levels of gold at $1.0 \mathrm{~V}$, which is depicted with a dotted square including the inactive redox center in Fig. 4g.

The energy levels of the oxidation of $A \beta$ residues are likely located between those of the gold electrodes under bias voltages (e.g., $1.0 \mathrm{~V}$ ), as shown in Fig. 4c, although this does not take into account coupling effects contributed by chemical contacts between the molecular orbitals and the gold electrode. Thus, electron transport can occur in the forward direction $(V=1.0 \mathrm{~V})$ via the energy levels of redox-active residues in mono-A $\beta$ junctions, suggesting hopping conductance. At the reverse direction $(V=-1.0 \mathrm{~V})$, the energy levels of redox centers can hardly allow for transporting electrons. In contrast, oli-A $\beta$ s did not exhibit voltage-dependent transport characteristics, indicating that redox centers are unlikely to be involved in the transport mechanism (Fig. 4f), which was consistent with the electrochemical activity results (Fig. 2d). Nonetheless, the molecular size-dependent $J / V$ plots and strong coupling of mono- and oli-A $\beta$ to the gold electrode via MPA revealed electron tunneling traversing

(a)

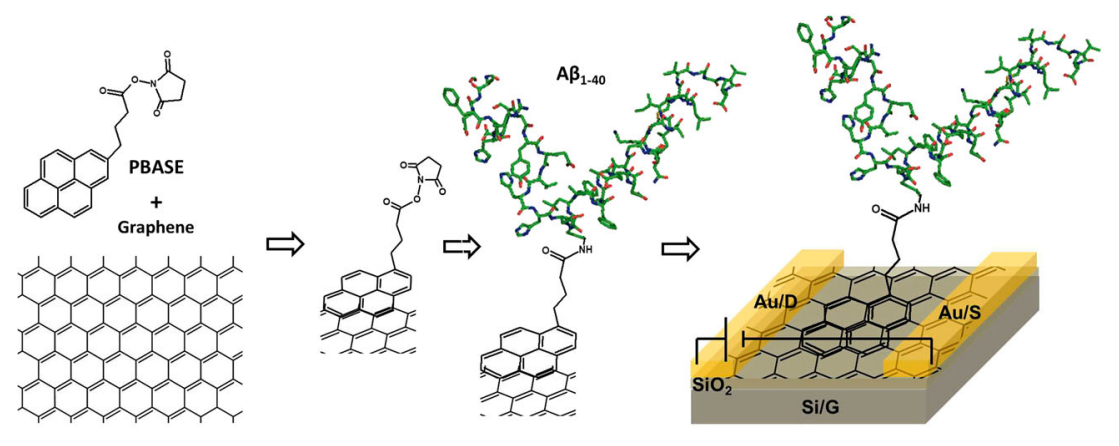

(b)

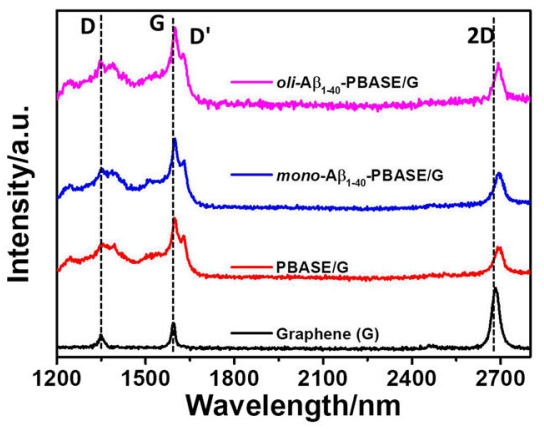

(c)

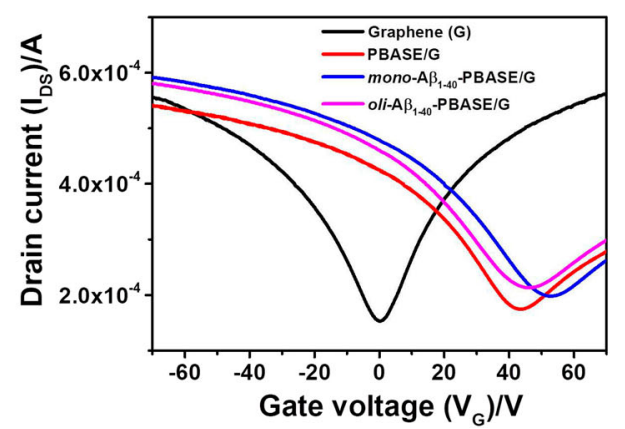

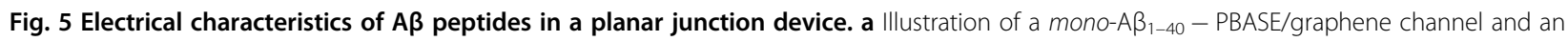
FET device. b Raman spectra of each graphene film on $\mathrm{SiO}_{2}$ substrates. $\mathbf{c}$ Electrical characteristics of each graphene FET device at $V_{D S}=0.1 \mathrm{~V}$. 
the energy barriers. Thus, at $V= \pm 1.0 \mathrm{~V}$, oli- $\mathrm{A} \beta \mathrm{s}$ can follow tunneling electron transport, while mono-A $\beta$ s can follow tunneling electron transport at $V=-1.0 \mathrm{~V}$ and the hopping electron transport at $V=1.0 \mathrm{~V}$.

\section{Electrical characteristics of $A \beta$ planar devices: $A \beta-P B A S E /$ graphene FETs}

Additionally, the electrical characteristics of a mono- or oli-A $\beta$ assembly in a planar device were explored. The A $\beta$-immobilized graphene channel was fabricated by using a self-assembled molecular linker monolayer of 1pyrenebutanoic acid succinimidyl ester (PBASE) (Fig. 5a). Transformation of mono-A $\beta$ s to oli-A $\beta$ s was conducted on mono-A $\beta$-immobilized surfaces under the same conditions for the formation of vertical oli-A $\beta$ junction devices. In the Raman fingerprint of graphene (Fig. 5b), a sharp and symmetric 2D band at approximately $2682.9 \mathrm{~cm}^{-1}$ was clearly observed and shifted to approximately $2695.5 \mathrm{~cm}^{-1}$ after modification with PBASE, indicating hole doping on pristine graphene. A D band at approximately $1348.0 \mathrm{~cm}^{-1}$ revealing structural defects on graphene appeared for both pristine graphene and PBASE-modified graphene. The G band of pristine graphene shifted from approximately $1595.0 \mathrm{~cm}^{-1}$ to approximately $1598.6 \mathrm{~cm}^{-1}$, indicating that the PBASE molecules induce hole doping on pristine graphene. A D' band appeared at approximately $1630.9 \mathrm{~cm}^{-1}$, indicating another weak disordered feature after modification with PBASE, which is superimposed with one of the peaks ${ }^{37}$ of the pyrene structure at $1242.2 \mathrm{~cm}^{-1}$, $1393.7 \mathrm{~cm}^{-1}$, and $1630.9 \mathrm{~cm}^{-1}$. The peak positions of the Raman bands were negligibly changed by further modification with mono-A $\beta$ s and oli-A $\beta$ s. The specific influence of mono- $\mathrm{A} \beta$ sequences was not observed in the peak positions of the Raman spectra (Fig. S7). In addition, the peak ratio of the intensity of the $G$ bands to $2 \mathrm{D}$ bands $\left(I_{\mathrm{G}} /\right.$ $I_{2 \mathrm{D}}$ ) increased with PBASE modification, indicating hole doping $^{37}$. The $I_{\mathrm{G}} / I_{2 \mathrm{D}}$ ratios increased with mono-A $\beta$ -PBASE modification and then decreased again with oliA $\beta$-PBASE modification.

The hole-doping effect of PBASE and A $\beta$ peptides on graphene was observed in the electrical characteristics of A $\beta$ - PBASE/G-FETs (Figs. $5 c$ and S7c, d). The pyrene linker PBASE on graphene induced a shift of the charge neutrality point to large positive values, indicating the highly concentrated doping of hole carriers. Then, the immobilization of mono-A $\beta$ s on PBASE/G increased the doping concentration of hole carriers produced by PBASE/G, and the charge neutrality point was positively shifted further. Furthermore, oligomerization of surface-tethered mono-A $\beta$ resulted in a negative shift of the charge neutrality point produced by mono-A $\beta-\mathrm{PBASE} / \mathrm{G}$. These phenomena were noticeably observed at all $\mathrm{A} \beta-\mathrm{PBASE} / \mathrm{G}-\mathrm{FETs}$ and were presumably associated with an increase in the surface hydrophobicity (or a decrease in the hydrophilic redox activity) due to the buried redox-active residues. This result was in agreement with the change in the water contact angle after the oligomerization of $A \beta$. The longer $A \beta$ peptide led to a larger shift in the charge neutrality point by both mono- and oli-modifications. Unlike oli-A $\beta$ s in vertical junctions, nonetheless, the electrical characteristics of oli-A $\beta-\mathrm{PBASE} / \mathrm{G}-\mathrm{FETs}$ according to peptide sequences were not distinguishable from those of mono-A $\beta-$ PBASE/G-FETs. The bottom line shows that both $A \beta$ vertical junctions and $A \beta / G$ FETs (especially $A \beta_{1-40}$ ) can provide new insights into understanding the electrical characteristics of oligomeric peptides discriminated from those of monomeric peptides, correlating with their electron transfer characteristics.

\section{Conclusion}

The electrical characteristics of surface-tethered monoand oli-A $\beta$ peptides were investigated. The structural characteristics of the $A \beta$ and the corresponding redox properties were significantly changed by oligomerization on surfaces. The electron transfer of $A \beta$ hardly proceeded after surface oligomerization; this observation was strongly related to a change in the electron transport pathway through $A \beta$. Different conformations of $A \beta_{1-40}$, $A \beta_{1-28}$, and $A \beta_{12-28}$ were used to explore the electrical characteristics of surface-tethered $A \beta$ peptides in two types of devices (e.g., vertical junction devices and graphene FET devices). In the vertical junction device, the electron transport pathway across the top gold-MPA// mono-A $\beta-\mathrm{MPA}$-bottom gold junctions was asymmetric with a strong dependence on the bias voltage. The redoxactive peptide residues of the mono-A $\beta$ s were strongly coupled with the bottom electrode through an MPA monolayer linker, which induced asymmetric coupling of the mono-A $\beta$ with gold electrodes at a high voltage (e.g., $1.0 \mathrm{~V})$. This coupling led to electron transport via the energy levels of redox centers through a hopping process. In contrast, the oli-A $\beta$ junctions exhibited symmetric characteristics due to reduced redox activity. The less redox-reactive molecular oli-A $\beta$ was symmetrically coupled with the electrode contacts, where the energy levels of redox centers were not positioned between the Fermi levels of gold electrodes and the electron transport could follow a tunneling process. However, the current flowing through the FETs with an A $\beta-P B A S E / G$ channel was weakly dependent on the peptide sequences and oligomerization. Consequently, investigation of sequencedependent electron transfer and transport characteristics of $A \beta$ peptides in vertical junctions can predict the chemical activity of ordered aggregates and distinguish the electron transport mechanism of oli-A $\beta$ peptides from that of mono-A $\beta$ peptides. 


\section{Materials and methods Materials}

Amyloid beta (A $\beta$ ) 1-40 human (Sigma-Aldrich, USA), $\left[\mathrm{Glu}^{11}\right.$ ] A $\beta$ 1-28 human (Aldrich, USA), and A $\beta$ 12-28 human (Sigma-Aldrich, USA) were purchased and used directly without further purification. Lyophilized $A \beta$ peptides were stored at $-20^{\circ} \mathrm{C}$. For the experiments, peptides were dissolved in $10 \mathrm{mM} \mathrm{PB} \mathrm{(pH} \mathrm{7.4,} \mathrm{purchased}$ from Sigma-Aldrich, USA) containing $1 \%$ ammonium hydroxide $\left(\mathrm{NH}_{4} \mathrm{OH}\right)$ solution $\left(80 \mu \mathrm{L}\right.$ of $1 \% \mathrm{NH}_{4} \mathrm{OH}$ solution was used for $1 \mathrm{mg}$ of $A \beta$ peptides) to obtain a final concentration of $25 \sim 50 \mu \mathrm{M} A \beta$. After vortexing for a few seconds, these solutions were used immediately.

\section{Fabrication of $A \beta$ peptide-MPA SAM-gold (or indium tin oxide)}

A SAM of MPA (Sigma-Aldrich) on a pre-cleaned gold/ silicon oxide $\left(\mathrm{Au} / \mathrm{SiO}_{2}\right)$, an $\mathrm{Au}(111)$ disc, or indium tin oxide on glass (ITO/glass) electrodes was formed in an ethanol solution containing $2 \mathrm{mM}$ MPA by incubation for $12 \mathrm{~h}$ at $4{ }^{\circ} \mathrm{C}$. The MPA SAM was washed thoroughly and dried, and then activated with 1-ethyl-3-(3-dimethylamino) propyl carbodiimide/ $N$-hydroxysuccinimide (EDC/NHS) in deionized (DI) water solution containing $10 \mathrm{mM} \mathrm{EDC/}$ NHS at $4{ }^{\circ} \mathrm{C}$ for $12 \mathrm{~h}$. After washing and drying the (EDC/ NHS)-MPA SAMs with ethanol and inert gas, respectively, a $50 \mu \mathrm{L}$ droplet of each $\mathrm{A} \beta$ peptide solution was dropped on the (EDC/NHS)-MPA SAM adsorbed substrates for $A \beta$ modification. These samples were placed into a $50 \mathrm{~mL}$ glass vial that was sealed to prevent water evaporation and incubated at $4{ }^{\circ} \mathrm{C}$ for $6-12 \mathrm{~h}$. Then, the A $\beta$-MPA SAMs were washed with PB and DI water and dried with inert gas. To fabricate the oli-A $\beta$-MPA SAMs, a $50 \mu \mathrm{L}$ droplet of $\mathrm{PB}$ solution containing $0.15 \mathrm{M}$ sodium chloride ( $\mathrm{NaCl}$, Sigma-Aldrich, USA) was dropped on the A $\beta$-MPA SAM substrates, and the substrates were placed into a $50 \mathrm{~mL}$ glass vial. The vial was sealed to prevent water evaporation and then incubated at $37^{\circ} \mathrm{C}$ in an incubator for a predetermined period of time (e.g., 1-4h). The wellwashed and dried mono-A $\beta-$ MPA or oli-A $\beta-$ MPA samples were stored in a vacuum desiccator in the dark.

\section{Fabrication of $A \beta$ vertical junction devices}

A cross-bar device for $A \beta$ vertical junctions was fabricated as reported in previous works ${ }^{30,35}$. Thin-film polydimethylsiloxane (PDMS)-coated polyethylene terephthalate (PET) substrates were used to pattern gold electrodes. PDMS solution was spin-coated on cleaned PET substrates and dried in a vacuum oven at $80^{\circ} \mathrm{C}$ overnight. Gold electrodes were patterned on PDMS/PET substrates by electron-beam (e-beam) deposition of gold using a shadow mask at a slow evaporation rate. Au electrode-patterned PDMS substrates were treated with oxygen plasma to become hydrophilic just before chemical modification.
The reliability test of the devices was performed by using different alkanethiol SAMs after device fabrication via top//bottom contact assembly (top electrode//molecular SAM-bottom electrode) as described in our previous report ${ }^{30}$. For the device fabrication of $A \beta$ vertical junction devices, a top electrode-patterned substrate modified with an MPA SAM was gently placed onto a bottom electrodepatterned substrate modified with an A $\beta-$ MPA SAM, and the top and bottom electrode arrays were aligned to form vertical junctions (top gold-MPA//A $\beta-M P A-b o t t o m$ gold) (Fig. S3).

\section{Fabrication of $A \beta$ peptide-PBASE/graphene FETs}

Graphene (SAINT, Sungkyunkwan University) was grown by chemical vapor deposition (CVD) on $\mathrm{Cu}$ foil and transferred onto $\mathrm{SiO}_{2}$ substrates with patterned gold electrodes by a polymethyl methacrylate (PMMA)-mediated method ${ }^{38}$. The transferred graphene was characterized by Raman spectroscopy with an excitation energy of $532 \mathrm{~nm}$ (Ntegra Spectra DUO Max, NT-MDT II). A graphene FET device was immersed in dimethylformamide (DMF) solution containing $10 \mathrm{mM}$ 1-pyrenebutanoic acid succinimidyl ester (PBASE, Aldrich) for the formation of $\pi-\pi$ stacked PBASE/graphene (PBASE/G) films at $4{ }^{\circ} \mathrm{C}$ for over $7 \mathrm{~h}$. The washed and dried PBASE/G FETs were used for the formation of mono-A $\beta$ peptide-PBASE/G and oliA $\beta$ peptide-PBASE/G as described above.

\section{Characterization of molecular assembly and devices}

Monomers and oligomers of $A \beta$ peptides were characterized by CD spectrometry (J-1500 CD spectrometer, Jasco, USA). Oligomerization of amyloid beta solution in $0.01 \mathrm{M} \mathrm{PB}$ was conducted by adding $\mathrm{NaCl}$ to a final concentration of $0.15 \mathrm{M}$ and incubation at $37^{\circ} \mathrm{C}$ for various times. For $\mathrm{CD}$ measurements of $\mathrm{A} \beta$ films, cleaned quartz substrates were used. Mono-A $\beta$ film on a quartz substrate was prepared by $6 \mathrm{~h}$ incubation at $4{ }^{\circ} \mathrm{C}$ after dropping a $50 \mu \mathrm{L}$ droplet of $\mathrm{PB}$ solution containing mono$\mathrm{A} \beta$ in a sealed vial. An oli-A $\beta$ film on a quartz substrate was prepared by $1 \mathrm{~h}$ incubation at $37^{\circ} \mathrm{C}$ after dropping a $50 \mu \mathrm{L}$ droplet of $\mathrm{PB}$ solution containing mono-A $\beta$ and $0.15 \mathrm{M} \mathrm{NaCl}$ in a sealed vial. Then, $A \beta$ samples were washed with $\mathrm{PB}$ and DI water and dried with inert gas.

Gold $/ \mathrm{SiO}_{2}$ substrates were used for the morphology and thickness analysis of $\mathrm{A} \beta$ films. Morphology and thickness measurements of three monomeric $A \beta$ films were conducted by AFM (SPM 5200, Agilent, USA) and ellipsometry (MG-1000, Nano-View, Korea). Morphological changes as a result of $A \beta$ oligomerization were verified by SEM (JSM-7100F, JEOL, USA). HOPG was used for the electrochemical study of physically adsorbed $A \beta$ films. The first few layers of HOPG were peeled off using tape and then used immediately. For physically adsorbed films of mono- or oli-A $\beta$ s to a certain substrate, a $50 \mu \mathrm{L}$ droplet 
of each solution was dropped on cleaned substrates, which were then placed into a $50 \mathrm{~mL}$ glass vial that was then sealed to prevent water evaporation and incubated at $4{ }^{\circ} \mathrm{C}$ for $6-12 \mathrm{~h}$. Then, $\mathrm{A} \beta$ samples were washed with $\mathrm{PB}$ and DI water and dried with inert gas.

Changes in surface binding energy as a result of chemical modification were verified by UPS (AXIS Supra, Kratos, UK). Water contact angle measurements (Phoenix-300, SEO, Korea) were performed for surface characterization of hydrophilicity and hydrophobicity. An Au (111) disc (MaTeck, Germany) was used for STM (SPM 5200, Agilent, USA) studies of A $\beta$-modified surfaces.

The $\mathrm{Au}(111)$ disc was chemically cleaned using piranha solution (a mixture of $\mathrm{H}_{2} \mathrm{SO}_{4}$ and $\mathrm{H}_{2} \mathrm{O}_{2}$ at a volume ratio of $3: 1$ ), thoroughly washed with DI water, and dried with inert gas. Then, the $\mathrm{Au}(111)$ disc was annealed by a hydrogen flame, cooled to room temperature, and immediately further chemically modified. The $\mathrm{A} \beta$-modified $\mathrm{Au}(111)$ sample as a working electrode was placed into an electrochemical STM cell composed of a Pt-wire counter electrode and a reference electrode. All electrochemical STM experiments were performed in a degassed aqueous solution containing $0.1 \mathrm{M} \mathrm{HClO}_{4}$ under inert gas.

Electrochemical studies of $A \beta$ molecules were performed by DPV (CHI 620, CH Instruments, USA) using a $5 \mathrm{~s}$ pulse period, $0.05 \mathrm{~V}$ amplitude, and $0.2 \mathrm{~s}$ pulse width. HOPG and ITO/glass electrodes were used for physically adsorbed $A \beta$ layers and chemically adsorbed $A \beta$ layers, respectively. A three-electrode electrochemical cell was used; Pt wire was used as the counter electrode, and an $\mathrm{Ag} \mid \mathrm{AgCl}$ (saturated $\mathrm{KCl}$ ) electrode was used as the reference electrode. The electrolyte was $0.1 \mathrm{M}$ PB solution degassed with an inert gas before use. The geometric area of the working electrodes was $0.2 \mathrm{~cm}^{2}$.

Current-voltage measurements of the devices were performed under vacuum $\left(\sim 1.0 \times 10^{-3}\right.$ torr $)$ at room temperature (Keithley 4200-SCS, Tektronix/Keithley, USA).

\section{Acknowledgements}

This work was supported by the National Research Foundation of Korea (NRF) grant funded by the Korea Government (MSIT) (NRF-2018R1A2B6006721) and IBS-R011-D1 and partially by a Korea Medical Device Development Fund grant funded by the Korean government (the Ministry of Science and ICT; the Ministry of Trade, Industry and Energy; the Ministry of Health \& Welfare; and the Ministry of Food and Drug Safety) (Project Number: KMDF_PR_20200901_0004).

\footnotetext{
Author details

'Department of Chemistry, Sungkyunkwan University (SKKU), Suwon 16419 Republic of Korea. ${ }^{2}$ Creative Research Institute (CRI), Sungkyunkwan University (SKKU), Suwon 16419, Republic of Korea. ${ }^{3}$ Centre for Integrated Nanostructure Physics (CINAP), Institute of Basic Science (IBS), Suwon 16419, Republic of Korea. ${ }^{4}$ Department of Biophysics, Sungkyunkwan University (SKKU), Suwon 16419, Republic of Korea
}

\section{Competing interests}

The authors declare no competing interests.

\section{Publisher's note}

Springer Nature remains neutral with regard to jurisdictional claims in published maps and institutional affiliations.

Supplementary information The online version contains supplementary material available at https://doi.org/10.1038/s41427-021-00321-z.

Received: 20 January 2021 Revised: 12 May 2021 Accepted: 4 June 2021. Published online: 16 July 2021

\section{References}

1. Marcus, R. A. \& Sutin, N. Electron transfers in chemistry and biology. Biochim. Biophys. Acta 811, 265-322 (1985).

2. Ron, I. et al. Proteins as electronic materials: electron transport through solid-state protein monolayer junctions. J. Am. Chem. Soc. 132, 4131-4140 (2010).

3. Amdursky, N. et al. Electronic transport via proteins. Adv. Mater. 26, 7142-7161 (2014).

4. Ing, N. L., El-Naggar, M. Y. \& Hochbaum, A. I. Going the distance: long-range conductivity in protein and peptide bioelectronic materials. J. Phys. Chem. B 122, 10403-10423 (2018).

5. Venkat, A. S., Corni, S. \& Di Felice, R. Electronic coupling between azurin and gold at different protein/substrate orientations. Small 3, 1431-1437 (2007).

6. Castañeda Ocampo, O. E. et al. Mechanism of orientation-dependent asymmetric charge transport in tunneling junctions comprising photosystem I. J. Am. Chem. Soc. 137, 8419-8427 (2015).

7. Fereiro, J. A. et al. Protein electronics: chemical modulation of contacts control energy level alignment in gold-azurin-gold junctions. J. Am. Chem. Soc. 140, 13317-13326 (2018)

8. Hamley, I. W. The amyloid beta peptide: a chemist's perspective. Role in Alzheimer's and fibrillization. Chem. Rev. 112, 5147-5192 (2012).

9. Rauk, A. The chemistry of Alzheimer's disease. Chem. Soc. Rev. 38, 2698-2715 (2009).

10. Chen, G.f et al. Amyloid beta: Structure, biology and structure-based therapeutic development. Acta Pharmacol. Sin. 38, 1205-1235 (2017).

11. Ahmed, M. et al. Structural conversion of neurotoxic amyloid- $\beta 1-42$ oligomers to fibrils. Nat. Struct. Mol. Biol. 17, 561-567 (2010).

12. Morel, B., Carrasco, M. P., Jurado, S., Marco, C. \& Conejero-Lara, F. Dynamic micellar oligomers of amyloid beta peptides play a crucial role in their aggregation mechanisms. Phys. Chem. Chem. Phys. 20, 20597-20614 (2018).

13. Ono, K., Condron, M. M. \& Teplow, D. B. Structure-neurotoxicity relationships of amyloid $\beta$-protein oligomers. Proc. Natl Acad. Sci. USA 106, 14745-14750 (2009).

14. Miller, Y., Ma, B. \& Nussinov, R. Polymorphism in Alzheimer a $\mathrm{\beta}$ amyloid organization reflects conformational selection in a rugged energy landscape. Chem. Rev. 110, 4820-4838 (2010).

15. Amit, M., Cheng, G., Hamley, I. W. \& Ashkenasy, N. Conductance of amyloid $\beta$ based peptide filaments: structure-function relations. Soft Matter 8, 8690-8696 (2012).

16. Heo, C. et al. Identifying fibrillization state of a $\beta$ protein via near-field thz conductance measurement. ACS Nano 14, 6548-6558 (2020).

17. Lee, S. J. C., Nam, E., Lee, H. J., Savelieff, M. G. \& Lim, M. H. Towards an understanding of amyloid- $\beta$ oligomers: characterization, toxicity mechanisms, and inhibitors. Chem. Soc. Rev. 46, 310-323 (2017).

18. Cohen, S. I. A. et al. Distinct thermodynamic signatures of oligomer generation in the aggregation of the amyloid- $\beta$ peptide. Nat. Chem. 10, 523-531 (2018).

19. Shea, D. et al. A-sheet secondary structure in amyloid $\beta$-peptide drives aggregation and toxicity in alzheimer's disease. Proc. Natl Acad. Sci. USA 116, 8895-8900 (2019)

20. Lindström, F., Bokvist, M., Sparrman, T. \& Gröbner, G. Association of amyloid- $\beta$ peptide with membrane surfaces monitored by solid state nmr. Phys. Chem. Chem. Phys. 4, 5524-5530 (2002).

21. Danielsson, J., Jarvet, J., Damberg, P. \& Gräslund, A. The alzheimer $\beta$-peptide shows temperature-dependent transitions between left-handed 31-helix, $\beta$-strand and random coil secondary structures. FEBS J. 272, 3938-3949 (2005).

22. Enache, T. A. \& Oliveira-Brett, A. M. Alzheimer's disease amyloid beta peptides in vitro electrochemical oxidation. Bioelectrochemistry 114, 13-23 (2017). 
23. Enache, T. A., Chiorcea-Paquim, A.-M. \& Oliveira-Brett, A. M. Amyloid- $\beta$ peptides time-dependent structural modifications: AFM and voltammetric characterization. Anal. Chim. Acta 926, 36-47 (2016).

24. Moores, B., Drolle, E., Attwood, S. J., Simons, J. \& Leonenko, Z. Effect of surfaces on amyloid fibril formation. PLoS ONE 6, e25954-e25954 (2011).

25. Vilan, A., Aswal, D. \& Cahen, D. Large-area, ensemble molecular electronics: motivation and challenges. Chem. Rev. 117, 4248-4286 (2017).

26. Wang, Z. et al. Role of redox centre in charge transport investigated by novel self-assembled conjugated polymer molecular junctions. Nat. Commun. 6, 7478 (2015).

27. Ruiz, M. P. et al. Bioengineering a single-protein junction. J. Am. Chem. Soc 139, 15337-15346 (2017).

28. Zhang, B., Song, W., Brown, J., Nemanich, R. \& Lindsay, S. Electronic conductance resonance in non-redox-active proteins. J. Am. Chem. Soc. 142, 6432-6438 (2020).

29. Kumar, K. S., Pasula, R. R., Lim, S. \& Nijhuis, C. A. Long-range tunneling processes across ferritin-based junctions. Adv. Mater. 28, 1824-1830 (2016).

30. Seo, S. et al. Nanoparticle linker-controlled molecular wire devices based on double molecular monolayers. Small 15, 1901183 (2019).

31. Yuan, L. et al. Controlling the direction of rectification in a molecular diode. Nat. Commun. 6, 6324 (2015)
32. Nijhuis, C. A., Reus, W. F. \& Whitesides, G. M. Mechanism of rectification in tunneling junctions based on molecules with asymmetric potential drops. J. Am. Chem. Soc. 132, 18386-18401 (2010).

33. Marques-Gonzalez, S. \& Low, P. Molecular electronics: history and fundamentals. Aust. J. Chem. 69, 244-253 (2016).

34. Lu, Q. et al. From tunneling to hopping: a comprehensive investigation of charge transport mechanism in molecular junctions based on oligo(p-phenylene ethynylene)s. ACS Nano 3, 3861-3868 (2009).

35. Seo, S., Hwang, E., Cho, Y., Lee, J. \& Lee, H. Functional molecular junctions derived from double self-assembled monolayers. Angew. Chem. Int. Ed. 56, 12122-12126 (2017)

36. He, J., Fu, Q., Lindsay, S., Ciszek, J. W. \& Tour, J. M. Electrochemical origin of voltage-controlled molecular conductance switching. J. Am. Chem. Soc. 128, 14828-14835 (2006)

37. Pantelic, R. S., Fu, W., Schoenenberger, C. \& Stahlberg, H. Rendering graphene supports hydrophilic with non-covalent aromatic functionalization for transmission electron microscopy. Appl. Phys. Lett. 104, 134103 (2014).

38. Seo, S., Min, M., Lee, S. M. \& Lee, H. Photo-switchable molecular monolayer anchored between highly transparent and flexible graphene electrodes. Nat. Commun. 4, 1920 (2013). 